\title{
Deviation from the $\operatorname{sech}^{2}$ superradiant emission law in a two-level atomic system
}

\author{
A. Edson Gonçalves \\ Departamento de Física, Fundação Universidade Estadual de Londrina, Londrina, 86051 PR, Brazil \\ Salomon S. Mizrahi \\ Departamento de Física, Universidade Federal de São Carlos, Via Washington Luiz, Km 235, \\ São Carlos, 13560 SP, Brazil \\ B. M. Pimentel* \\ Instituto de Física Teórica, UNESP, Rua Pamplona, 145, São Paulo, 01405 SP, Brazil
}

(Received 29 May 1990)

\begin{abstract}
The atomic superradiant emission is treated in the single-particle mean-field approximation. A single-particle Hamiltonian, which represents a dressed two-level atom in a radiation field, can be obtained and it is verified that it describes the transient regime of the emission process. While the line-shape emission for a bare atom follows the $\operatorname{sech}^{2}$ law, for the dressed atom the line shape deviates appreciably from this law and it is verified that the deviation depends crucially on the ratio of the dynamic frequency shift to the transition frequency. This kind of deviation is observed in experimental results.
\end{abstract}

Since the theoretical prediction of the superradiance (SR) phenomenon by Dicke in 1954 (Ref. 1) and the first experimental observation performed by Skribanowitz et al. in 1973 (Ref. 2) a lot of work has been devoted to the subject, ${ }^{3-10}$ in the experimental as in the theoretical areas. The superradiant emission consists essentially in the spontaneous collective emission of an electromagnetic (EM) pulse that takes place in a moderately dense system composed by $N$ two-level atoms (or molecules). The atoms are prepared in an initial population inversion state which reverts, spontaneously, to the ground state in a time inversely proportional to the number of atoms, $\tau_{c} \propto N^{-1}$, such that the emission intensity turns out to be proportional to $N^{2}$. The SR pulse occurs due to the induced correlations that develop among the transition dipole moments of the atoms as they interact with each other, not directly, but through the coherent radiation they emit.

The SR phenomenon turns out to be simpler than the process of the laser emission because one can ignore the pumping and relaxation mechanism of the walls, it is sufficient to consider the evolution of the atoms coupled exclusively to their own radiation field. Several approaches have been advanced toward a theoretical description of the SR, all of them arrive either to a master equation for the atomic system (Schrödinger picture) or to a set of coupled first-order differential equations, the Maxwell-Bloch equations (Heisenberg picture). Furthermore several approximations are introduced in order to obtain physically transparent analytical expressions. Among these one can cite the Born-Markov approximation, the rotating-wave approximation (RWA), the small sample size assumption (the sample size is smaller than the wavelength of the emitted radiation) or the large cylindrical pencil-shaped sample model (the linear dimen- sion $L$, is much larger than both the wavelength $\lambda$ and the transverse dimension $d$ satisfying the relation $L \gg \lambda \gg d$ ).

The full Hamiltonian of the radiation-matter system is assumed to be composed by three terms,

$$
H=H_{S}+H_{R}+V
$$

with

$$
\begin{aligned}
& H_{S}=\omega_{0} \sum_{i} s_{0}(i) \\
& H_{R}=\sum_{n} \omega_{n} b_{n}^{\dagger} b_{n}, \\
& V=\sum_{n, i} \kappa_{n}\left[b_{n}^{\dagger} s_{-}(i)+b_{n} s_{+}(i)\right],
\end{aligned}
$$

where $H_{S}$ is the Hamiltonian of the emitters (bare atoms), $\omega_{0}$ is the transition frequency of the two-level atoms, $H_{R}$ is the Hamiltonian of the radiation field, and $V$ is the matter-radiation interaction considered in the RWA, where $\kappa_{n}$ stands for the radiation-matter coupling parameter.

Considering that the radiation field acts like a heat bath that surrounds the atomic system, this one evolves irreversibly from an initial to a final state with the evolution governed by a master equation for the reduced statistical operator. In the one-body mean-field approximation the atomic master equation is ${ }^{9-12}$

$$
\begin{aligned}
\dot{\rho}= & -i[\mathcal{H}(\rho), \rho] \\
& -\frac{\gamma}{2}\left[\bar{n}\left(s_{-} s_{+} \rho-2 s_{+} \rho s_{-}+\rho s_{-} s_{+}\right)\right. \\
& \left.\quad+(\bar{n}+1)\left(s_{+} s_{-} \rho-2 s_{-} \rho s_{+}+\rho s_{+} s_{-}\right)\right],
\end{aligned}
$$


where

$$
\begin{aligned}
\mathcal{H}(\rho)= & \bar{\omega} s_{0}+(N-1) \Delta \omega\left(s_{-}\left\langle s_{+}\right\rangle+s_{+}\left\langle s_{-}\right\rangle\right) \\
& +i(N-1) \frac{\gamma}{2}\left(s_{-}\left\langle s_{+}\right\rangle-s_{+}\left\langle s_{-}\right\rangle\right) \\
= & \bar{\omega} s_{z}+(N-1)\left[\left(2 \Delta \omega\left\langle s_{x}\right\rangle-\gamma\left\langle s_{y}\right\rangle\right) s_{x}+\left(2 \Delta \omega\left\langle s_{y}\right\rangle\right.\right. \\
& \left.\left.+\gamma\left\langle s_{x}\right\rangle\right) s_{y}\right]
\end{aligned}
$$

is the single-particle mean-field Hamiltonian, which describes a dressed atom, and \langle\rangle stands for mean value. The parameters that enter the master equation (3) are

$$
\bar{\omega}=\omega_{0}+\Delta \omega
$$

where

$$
\left.\Delta \omega=-\frac{\gamma}{2 \pi} \ln \left[\mid \frac{\omega_{c}}{\omega_{0}}+1\right]\left|\frac{\omega_{c}}{\omega_{0}}-1\right|\right]
$$

is the dynamical frequency shift, $\gamma$ is the spontaneous decay constant, and $\omega_{c}$ is a cutoff frequency, while

$$
\bar{n}=\frac{1}{\exp \left(\hbar \omega_{0} / k_{B} T\right)-1}
$$

is the mean photon number.

The master equation permits one to write the time derivative of the spin components ${ }^{13}$ mean values,

$$
\begin{aligned}
\left\langle s_{x}\right\rangle= & -\bar{\omega}\left\langle s_{y}\right\rangle-\gamma\left(\bar{n}+\frac{1}{2}\right)\left\langle s_{x}\right\rangle+(N-1) \gamma\left\langle s_{x}\right\rangle\left\langle s_{z}\right\rangle \\
& +2(N-1) \Delta \omega\left\langle S_{y}\right\rangle\left\langle S_{z}\right\rangle, \\
\left\langle s_{y}\right\rangle= & \bar{\omega}\left\langle s_{x}\right\rangle-\gamma\left(\bar{n}+\frac{1}{2}\right)\left\langle s_{y}\right\rangle+(N-1) \gamma\left\langle s_{y}\right\rangle\left\langle s_{z}\right\rangle \\
& -2(N-1) \Delta \omega\left\langle s_{x}\right\rangle\left\langle s_{z}\right\rangle, \\
\left\langle s_{z}\right\rangle= & -\frac{1}{2} \gamma-2 \gamma\left(\bar{n}+\frac{1}{2}\right)\left\langle s_{z}\right\rangle \\
& -\gamma(N-1)\left(\left\langle s_{x}\right\rangle^{2}+\left\langle s_{y}\right\rangle^{2}\right),
\end{aligned}
$$

and one verifies that this system is not integrable because it has not any constant of motion, however for the meanfield Hamiltonian there is an invariant, the Bloch vector $\left\langle s_{x}\right\rangle_{s_{x}}+\left\langle s_{y}\right\rangle_{s_{y}}+\left\langle s_{z}\right\rangle_{s_{z}}$ which permits an exact solution of Schrödinger equation, $i \partial_{t} \psi=\mathscr{H}(\rho) \psi .^{12}$ As we have introduced the conjecture that the Hamiltonian (4) represents a dressed atom, then one defines the intensity of the radiation field (in units of $\hbar \bar{\omega}$ ) as

$$
\begin{aligned}
\mathcal{J}= & -\frac{N}{\gamma} \frac{d}{d t}\langle\mathcal{H}(\rho)\rangle \\
= & \frac{N}{2}+2\left(\bar{n}+\frac{1}{2}\right)\left\langle S_{z}\right\rangle \\
& +\left(1+4 \frac{\Delta \omega}{\bar{\omega}}\left(\bar{n}+\frac{1}{2}\right)-4 \frac{\Delta \omega}{\bar{\omega}}\left\langle S_{z}\right\rangle\right]\left(\left\langle S_{x}\right\rangle^{2}+\left\langle S_{y}\right\rangle^{2}\right)
\end{aligned}
$$

instead of the usual definition which makes use of the bare Hamiltonian. ${ }^{11}$ Here one has $\left\langle S_{x}\right\rangle=N\left\langle s_{x}\right\rangle$, $\left\langle S_{y}\right\rangle=N\left\langle s_{y}\right\rangle,\left\langle S_{z}\right\rangle=N\left\langle s_{z}\right\rangle$ and the set of equations (7) were used in order to introduce the dissipative terms in Eq. (8).

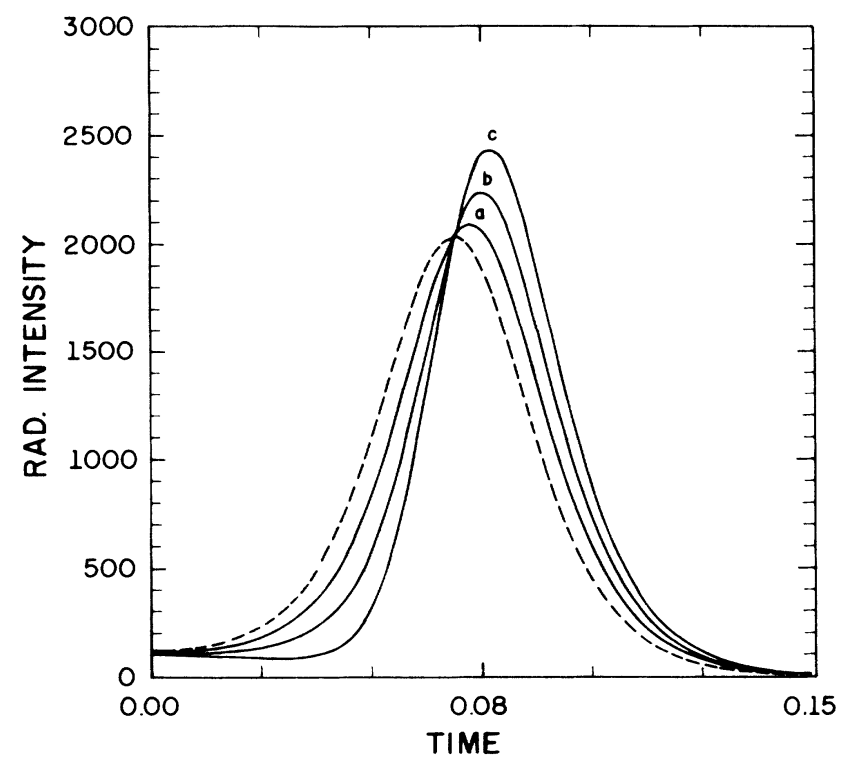

FIG. 1. The plot is of $\mathcal{I}$ vs $t$, for $\bar{n}=0$. The dashed curve corresponds to $\Delta \omega=0$ while the solid curves labeled by $a, b$, and $c$ are for the set of values $\Delta \omega / \bar{\omega}=2 \times 10^{-3}, 4 \times 10^{-3}$, and $6 \times 10^{-3}$, respectively. The time is given in units of the decay constant $\gamma$ while the intensity $\mathcal{J}$ in units of $\hbar \bar{\omega}$.

The intensity $\mathcal{J}$ and time $t$ are plotted for several values of $\Delta \omega / \bar{\omega}$ and for two values of $\bar{n}$. One has assumed $N=100$ and attributed values for $\Delta \omega / \bar{\omega}$ such that the condition $(\Delta \omega / \bar{\omega}) N<1$ be valid. ${ }^{12}$ In Fig. $1, \bar{n}=0$; the dashed curve corresponds to $\Delta \omega=0$; it is the case of considering the bare atom Hamiltonian $\mathcal{H}=\bar{\omega} s_{0}$; it preserves the symmetry of the $\operatorname{sech}^{2}$ emission law about $t_{0}$ (the delay time or time of maximum intensity emission). The

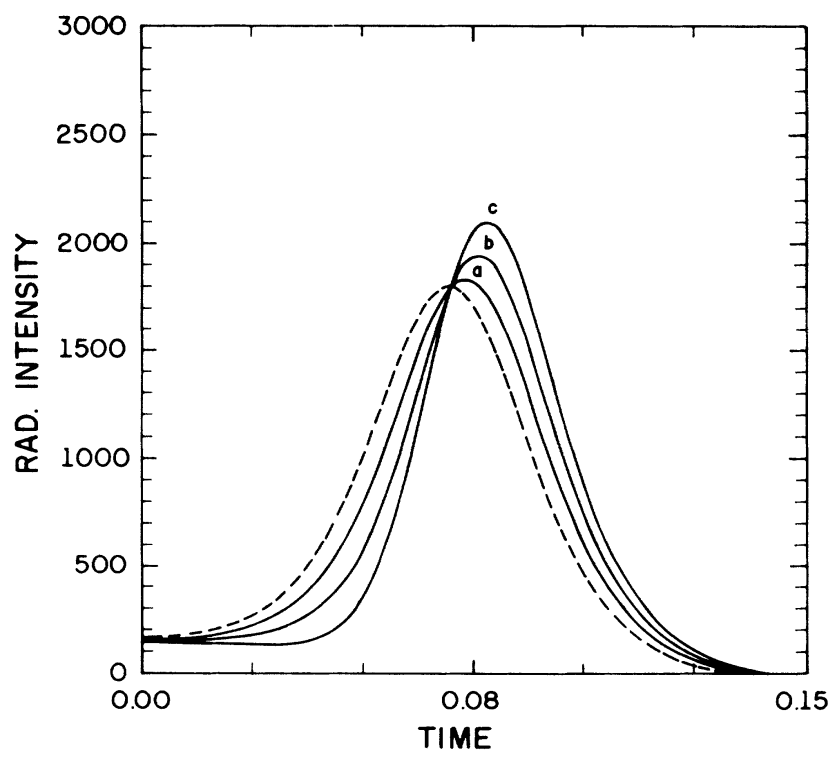

FIG. 2. The plot $\mathscr{I}$ vs $t$ is for $\bar{n}=0.5$. All the other parameters are the same as for Fig. 1. 
other curves labeled by $a, b$, and $c$ correspond respectively to $\Delta \omega / \bar{\omega}=2 \times 10^{-3}, 4 \times 10^{-3}$, and $6 \times 10^{-3}$, and their main features are the following.

(i) With deviation from the symmetry about the delay time, the slope of the curve is smoother on the right than on the left. On the other hand, the inverse behavior occurs if one considers $\Delta \omega<0$ (see Figs. 1 and 2 of Ref. 2).

(ii) The peak intensity is higher the greater the value of the ratio $\Delta \omega / \bar{\omega}$. This behavior occurs because the emission line becomes narrower whereas the area under the curve is conserved.

Identical conclusions are obtained for $\bar{n} \neq 0$ (see Fig. 2) and for the same set of values of $\Delta \omega / \bar{\omega}$; all the same here, the peak intensity is less, due to the effects of temperature that enhance the contribution of the dissipative terms over the ones responsible for loss of coherence, the commutator in Eq. (3).

In summary, using the dressed atom conjecture Eqs. (4) and (8), the line shapes deviates appreciably from the $\operatorname{sech}^{2}$ law and this feature is observed in experimental results, i.e., pulse signals, oscilloscope traces, etc. ${ }^{2-7}$ So the mean-field Hamiltonian, Eqs. (4a) or (4b), can explain a further aspect of the SR emission which manifests through the presence of the dynamic frequency shift in the emission intensity expression, Eq. (8).

S.S.M. and B.M.P. received partial financial support from the CNPq, Brazil.
*Electronic address: uesp@ brfapesp.bitnet.

${ }^{1}$ R. H. Dicke, Phys. Rev. 93, 99 (1954).

${ }^{2}$ N. Skribanowitz et al., Phys. Rev. Lett. 30, 309 (1973).

${ }^{3}$ M. Gross et al., Phys. Rev. Lett. 36, 1035 (1976).

${ }^{4}$ H. M. Gibbs et al., Phys. Rev. Lett. 39, 547 (1977).

${ }^{5}$ M. Gross et al., Phys. Rev. Lett. 40, 1711 (1978).

${ }^{6}$ A. Crubellier et al., Phys. Rev. Lett. 41, 1237 (1978).

${ }^{7}$ M. Gross et al., Phys. Rev. Lett. 43, 343 (1979).

${ }^{8}$ A. V. Andreev et al., Usp. Fiz. Nauk 131, 653 (1980) [Sov.
Phys.-Usp. 23, 493 (1980)].

${ }^{9}$ M. Gross and S. Haroche, Phys. Rep. 93, 301 (1982).

${ }^{10}$ C. Leonardi et al., Riv. Nuovo Cimento 9, 1 (1986).

${ }^{11}$ G. S. Agarwal, Quantum Statistical Theories of Spontaneous Emission, Vol. 70 of Springer Tracts in Modern Physics (Springer-Verlag, Berlin, 1984), and references therein.

${ }^{12}$ S. S. Mizrahi, Phys. Lett. A 144, 282 (1990).

${ }^{13}$ The algebraic isomorphism between operators of a spin- $\frac{1}{2}$ particle and a two-level atom has been used. 